Please do not remove this page

RMIT

UNIVERSITY

\title{
Reverse engineering of a fixed wing unmanned aircraft 6-DoF model for navigation and guidance applications
}

Burston, Martin; Sabatini, Roberto; Clothier, Reece; Gardi, Alessandro; Ramasamy, Subramanian https://researchrepository.rmit.edu.au/esploro/outputs/9921862290401341/filesAndLinks?institution=61RMIT_INST\&index=null

Burston, M., Sabatini, R., Clothier, R., Gardi, A., \& Ramasamy, S. (2014). Reverse engineering of a fixed wing unmanned aircraft 6-DoF model for navigation and guidance applications. Applied Mechanics and Materials, 629, 164-169. https://doi.org/10.4028/www.scientific.net/AMM.629.164 Document Version: Accepted Manuscript

Published Version: https://doi.org/10.4028/www.scientific.net/AMM.629.164

Repository homepage: https://researchrepository.rmit.edu.au

(C) 2014 Applied Mechanics and Materials

Downloaded On 2023/04/26 19:07:51 +1000

Please do not remove this page 
Thank you for downloading this document from the RMIT Research Repository.

The RMIT Research Repository is an open access database showcasing the research outputs of RMIT University researchers.

RMIT Research Repository: http://researchbank.rmit.edu.au/

\section{Citation:}

Burston, M, Sabatini, R, Clothier, R, Gardi, A and Ramasamy, S 2014, 'Reverse engineering of a fixed wing unmanned aircraft 6-DoF model for navigation and guidance applications', Applied Mechanics and Materials, vol. 629, pp. 164-169.

See this record in the RMIT Research Repository at:

https://researchbank.rmit.edu.au/view/rmit:24973

Version: Accepted Manuscript

Copyright Statement: (c) 2014 Applied Mechanics and Materials

Link to Published Version:

http://dx.doi.org/10.4028/www.scientific.net/AMM.629.164 
This is the author pre-publication version. This paper does not include the changes arising from the revision, formatting and publishing process. The final paper that should be used for referencing is:

M. Burston, R. Sabatini, R. Clothier, A. Gardi, S. Ramasamy, "Reverse Engineering of a Fixed Wing Unmanned Aircraft 6-DoF Model for Navigation and Guidance Applications", Applied Mechanics and Materials, vol. 629, pp. 164-169, Trans Tech Publications, 2014. DOI: 10.4028/www.scientific.net/AMM.629.164

\title{
Reverse Engineering of a Fixed Wing Unmanned Aircraft 6-DoF Model for Navigation and Guidance Applications
}

\author{
Martin T. Burston ${ }^{1}$, Roberto Sabatini ${ }^{1, a}$, Reece Clothier $^{1}$, \\ Alessandro Gardi ${ }^{1}$ and Subramanian Ramasamy ${ }^{1}$ \\ ${ }^{1}$ School of Aerospace, Mechanical and Manufacturing Engineering \\ RMIT University, Melbourne, VIC 3000, Australia \\ aroberto.sabatini@rmit.edu.au
}

Keywords: 6-DoF, Flight Dynamics, Laser Scanner, Navigation and Guidance System, Reverse Engineering and Unmanned Aircraft.

\begin{abstract}
A method for deriving the parameters of a six-degree-of-freedom (6-DoF) aircraft dynamics model by adopting reverse engineering techniques is presented. The novelty of the paper is the adaption of the 6-DoF Aircraft Dynamics Model (ADM) as a virtual sensor integrated in a low-cost navigation and guidance system designed for small Unmanned Aircraft (UA). The mass and aerodynamic properties of the JAVELIN UA are determined with the aid of an accurate 3D scanning and CAD processing. For qualitatively assessing the calculated ADM, a trajectory with high dynamics is simulated for the JAVELIN UA and compared with that of a published 6-DoF model of the AEROSONDE UA. Additionally, to confirm the validity of the approach, reverse engineering procedures are applied to a published CAD model of the AEROSONDE UA aiding to the calculation of the associated 6-DoF model parameters. A spiral descent trajectory is generated using both the published and calculated parameters of the AEROSONDE UA and a comparative analysis is performed that validates the methodology. The accurate knowledge of the ADM is then utilised in the development of a virtual sensor to augment the UA navigation and guidance system in case of primary navigation sensor outages.
\end{abstract}

\section{Introduction}

In the recent years, the use of Unmanned Aircraft (UA) in a number of civil and military applications is accelerating at an unprecedented rate. In this context, the six-degree-of-freedom (6DoF) Aircraft Dynamics Model (ADM) for an UA is essential for carrying out a host of research activities. This paper presents an approach for developing the 6-DoF dynamics model without any reference to original design drawings and manufacturer detained information. Reverse-engineering techniques are used to retrieve the detailed geometric information about the UA airframe, which are then made use of for replicating the mass properties of the UA [1]. The retrieved 6-DoF model is then implemented onboard the UA as a virtual sensor in a similar manner to that of proposed in [2] to compensate for the shortcomings of the Global Navigation Satellite System (GNSS) sensors and Micro-Electro-Mechanical System (MEMS) based Inertial Measurement Unit (IMU) in highdynamics attitude determination tasks. The term 'virtual sensor' is used to describe a system in which an ADM, in addition to GNSS, MEMS-IMU and Vision Based Navigation (VBN) sensors (employed for precision approach and landing phases), provides additional inputs to an Extended/ Unscented Kalman Filter (EKF/UKF) fusion and estimation architecture [2-5].

\section{Reverse Engineering Methodology}

The reverse engineering methodology presented in this paper involves development of a virtual model, or mockup, of the UA. The method uses 3D scanning techniques and computer-aided design (CAD) package for reconstructing the model. The mass properties of the UA are then determined from the virtual mockup in CAD. The derivation of the aerodynamic coefficients and derivatives is performed in parallel using point measures of the airframe. The dynamics model is finally validated 
This is the author pre-publication version. This paper does not include the changes arising from the revision, formatting and publishing process. The final paper that should be used for referencing is:

M. Burston, R. Sabatini, R. Clothier, A. Gardi, S. Ramasamy, "Reverse Engineering of a Fixed Wing Unmanned Aircraft 6-DoF Model for Navigation and Guidance Applications", Applied Mechanics and Materials, vol. 629, pp. 164-169, Trans Tech Publications, 2014. DOI: 10.4028/www.scientific.net/AMM.629.164

by first qualitatively comparing its simulated trajectory to that of a verified 6-DoF published in literature. Further quantitative verification and refinements are performed using flight and wind tunnel tests. For this research, we used the most recent addition to RMIT University UA research fleet, the "JAVELIN", a fixed wing UA. The parameters of the UA are listed in Table 1. Table 1 also lists the AEROSONDE Mk1 UA parameters for a comparative study [6, 7]. Additionally, Table 1 (right) lists the characteristics of the Handyscan EXAscan 3D laser scanner used to obtain 3D surface geometry data of the UA [8].

Table 1. Comparison of AEROSONDE Mk1 and JAVELIN UA parameters (left) and Handyscan EXAscan 3D scanner parameters (right).

\begin{tabular}{|c|c|c|}
\hline Parameter & AEROSONDE & JAVELIN \\
\hline Length [mm] & 1700 & 2000 \\
\hline Height [mm] & 600 & 650 \\
\hline Wingspan [mm] & 2900 & 2800 \\
\hline Aspect Ratio & 15 & 11 \\
\hline Empty Weight [kg] & 8.5 & 8.7 \\
\hline MTOW [kg] & 13.5 & $15+$ \\
\hline Fuel Capacity [L] & 5 & 2 \\
\hline Endurance [hours] & $30+$ & $\sim 3$ \\
\hline Engine [cc] & 20 & 20 \\
\hline Max Power [kW] & 1.28 & 1.28 \\
\hline
\end{tabular}

\begin{tabular}{|c|c|}
\hline $\begin{array}{c}\text { 3D Scanner } \\
\text { Parameter }\end{array}$ & Value \\
\hline Weight $[\mathrm{kg}]$ & 1.25 \\
\hline Dimensions [mm] & $172 \times 260 \times 216$ \\
\hline $\begin{array}{c}\text { Measurement Rate } \\
\text { [measures } / \mathrm{s}]\end{array}$ & 25,000 \\
\hline Laser Class & II (eye-safe) \\
\hline Resolution [mm] & 0.050 \\
\hline Accuracy [mm] & Up to 0.040 \\
\hline
\end{tabular}

In order to determine the relative position to the surfaces the scanned object required a covering of a random arrangement of reflective stickers (dots). The randomness of the placement of the dots was essential as the scanner relied on the unique patterns of groups of 4-5 dots in order to locate itself [9]. The point data obtained from the scanner were then processed into geometric surfaces using the reverse engineering software, Geomagic. The geometric surfaces obtained were then exported to the CAD program - CATIA V5. The 3D scan files provided accurate external geometry of the airframe - in most cases with less than $1 \%$ deviation from the true distances in the geometry, but no data was obtainable for internal structures due to the inaccessibility of those areas to the scanner Adopting the aforementioned methodology, three-view engineering drawings of the scanned files were obtained. The drawings were used as guidelines for reconstruction of the parts in CATIA as illustrated in Fig. 1.
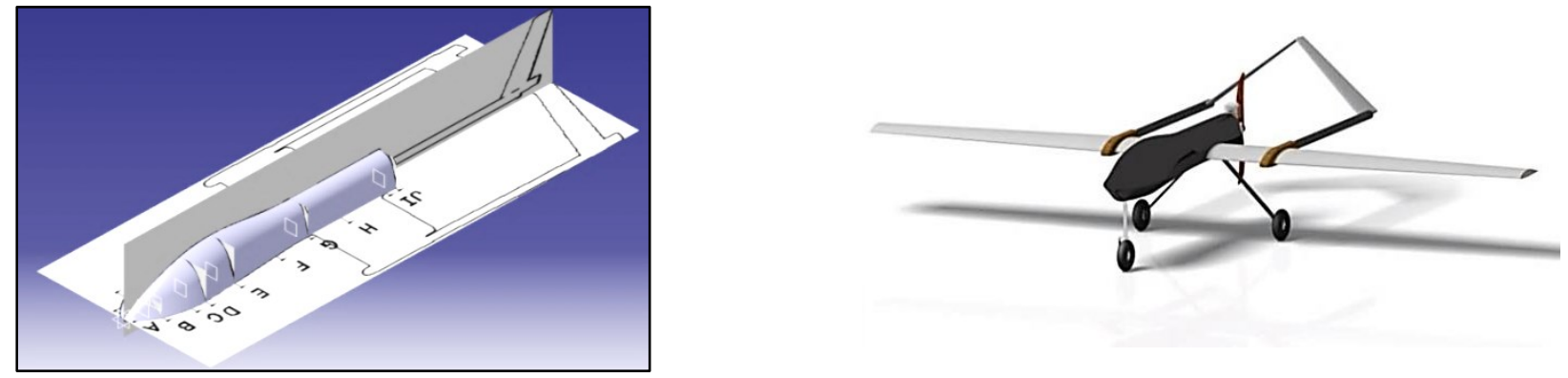

Fig. 1. Reconstruction of the fuselage in CATIA and the finalized 3D CAD of the JAVELIN UA.

The center of gravity ( $\mathrm{CoG}$ ) location and mass moments of inertia of the JAVELIN about its axes were determined using the assembly of all of the digitized components in CATIA. By splitting the components into sub-parts, an approximation of the distribution of mass throughout the airframe was performed. 
This is the author pre-publication version. This paper does not include the changes arising from the revision, formatting and publishing process. The final paper that should be used for referencing is:

M. Burston, R. Sabatini, R. Clothier, A. Gardi, S. Ramasamy, "Reverse Engineering of a Fixed Wing Unmanned Aircraft 6-DoF Model for Navigation and Guidance Applications", Applied Mechanics and Materials, vol. 629, pp. 164-169, Trans Tech Publications, 2014. DOI: 10.4028/www.scientific.net/AMM.629.164

\section{Development of the 6-DoF Dynamics Model}

A 6-DoF theoretical model was developed after obtaining the mass properties of the UA. The response of the UA to disturbances and control inputs was obtained based on the model. The 6-DoF dynamics model of the JAVELIN UA is derived using the equations of motion for a fixed-wing aircraft. For implementation purposes, the aerodynamic derivatives and coefficients are essential, which were determined using a number of techniques. The majority of stability and control derivatives were calculated using empirical equations originally developed for DATCOM as discussed in [9]. Sensitive coefficients such as zero-alpha lift and lift-curve slope were calculated using a 3D vortex lattice method in order to achieve higher accuracy for the given UA geometry. The JAVELIN model in XLR5 analysis tool after performing 3D vortex lattice analysis is illustrated in Fig. 2. A preliminary analysis of the calculated derivatives and coefficients was performed by comparing the calculated parameters of the JAVELIN with the validated parameters for a geometric and inertially similar UA - the AEROSONDE Mk1, the parameters for which are provided with the AeroSim Blockset for Simulink [6]. A comparison of several stability derivatives, separated into groups and ordered by importance by [10] are given in Table 2.

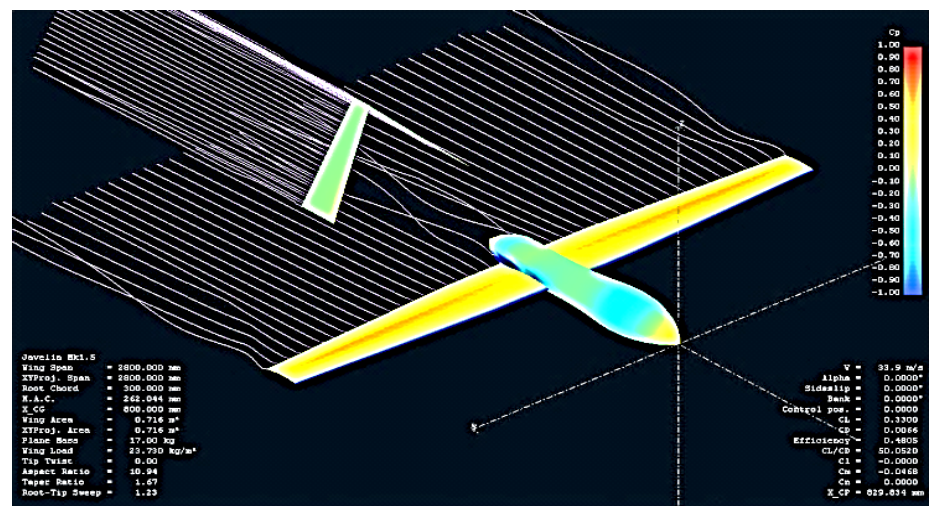

Fig. 2. The JAVELIN model in XFLR5 analysis tool.

Table 2. Stability derivatives and moments of inertia for AEROSONDE and JAVELIN UA [6, 10].

\begin{tabular}{|c|c|c|c|c|}
\hline Parameter & Units & AEROSONDE & JAVELIN & Determination Method \\
\hline$C_{L_{\alpha}}$ & $/ \mathrm{rad}$ & 5.61 & 4.97 & 3D Vortex Lattice Using [11] \\
\hline$C_{m_{\alpha}}$ & $/ \mathrm{rad}$ & -2.74 & -1.57 & 3D Vortex Lattice Using [1]] \\
\hline$C_{l_{\beta}}$ & $/ \mathrm{rad}$ & -0.13 & -0.073 & Empirical [10] \\
\hline$C_{n_{\beta}}$ & $/ \mathrm{rad}$ & 0.073 & 0.072 & Empirical [10] \\
\hline$C_{m_{\dot{\alpha}}}$ & $/ \mathrm{rad}$ & -10.38 & -2.05 & Empirical [10] \\
\hline$C_{m_{q}}$ & $/ \mathrm{rad}$ & -38.21 & -7.24 & Empirical [10] \\
\hline$C_{l_{p}}$ & $/ \mathrm{rad}$ & -0.51 & -0.46 & Empirical [10] \\
\hline$C_{n_{r}}$ & $/ \mathrm{rad}$ & -0.095 & -0.075 & Empirical [10] \\
\hline$C_{D_{m i n}}$ & $/ \mathrm{rad}$ & 0.043 & 0.03 & 3D Vortex Lattice Using [11] \\
\hline$C_{L_{0}}$ & $/ \mathrm{rad}$ & 0.23 & 0.33 & 3D Vortex Lattice Using [11] \\
\hline$C_{m_{0}}$ & $/ \mathrm{rad}$ & 0.135 & 0.135 & 3D Vortex Lattice Using [11] \\
\hline$C_{L_{\dot{\alpha}}}$ & $/ \mathrm{rad}$ & 1.97 & 0.62 & Empirical [10] \\
\hline$C_{L_{q}}$ & $/ \mathrm{rad}$ & 7.95 & 6.70 & Empirical [10] \\
\hline$I_{x x}$ & $\mathrm{kgm}^{2}$ & 0.824 & 1.647 & CAD Model \\
\hline$I_{y y}$ & $\mathrm{kgm}^{2}$ & 1.135 & 1.531 & CAD Model \\
\hline$I_{z Z}$ & $\mathrm{kgm}^{2}$ & 1.759 & 3.094 & CAD Model \\
\hline$I_{x z}$ & $\mathrm{kgm}^{2}$ & 0.120 & 0.066 & CAD Model \\
\hline
\end{tabular}


This is the author pre-publication version. This paper does not include the changes arising from the revision, formatting and publishing process. The final paper that should be used for referencing is:

M. Burston, R. Sabatini, R. Clothier, A. Gardi, S. Ramasamy, "Reverse Engineering of a Fixed Wing Unmanned Aircraft 6-DoF Model for Navigation and Guidance Applications", Applied Mechanics and Materials, vol. 629, pp. 164-169, Trans Tech Publications, 2014. DOI: 10.4028/www.scientific.net/AMM.629.164

In addition to the aerodynamic coefficients, the moments of inertia are required to complete describe the ADM. These are determined from the detailed CAD model developed earlier, and are compared to the values of the AEROSONDE. Significant similarities can be seen from Table 2.

\section{6-DoF ADM Validation}

In order to validate the JAVELIN 6-DoF ADM without performing flight tests, a comparative approach is considered, whereby an open-loop simulation is performed for the JAVELIN and the AEROSONDE UA. For simulation purposes, it is assumed that the propulsion model for the JAVELIN is the same as that of the AEROSONDE UA. The simulation begins with each aircraft at $250 \mathrm{~m}$ ASL (230 $\mathrm{m}$ AGL at the location of the simulation), with the throttle set to $40 \%$. Although the UA are trimmed for steady-level flight, without control inputs the unbalanced torque from the engine excites the spiral mode and the aircraft begin to descent in a helix trajectory. The comparison of the spiral trajectories obtained for the two UA are illustrated in Fig. 3 (a). Due to a greater bank angle and a shorter time to reach the ground, it can be seen that the JAVELIN completes fewer loops before it reaches the end point. Further qualitative verification of this method is undertaken by comparing the trajectories plotted using the aerodynamic derivatives of the AEROSONDE as published by Aerosim [6] and the same derivatives calculated from a CAD model of the AEROSONDE. The estimated $C_{n_{\beta}}$ appears significantly larger than the published value, and this can be effectively noticed in the spiral mode, which is sensitive to this parameter. Fig. 3 (b) illustrates the performance comparison of the calculated 6-DoF for the AEROSONDE UA to that of the published 6-DoF when lateral, longitudinal and airspeed closed-loop PI \& PID control methods are implemented.

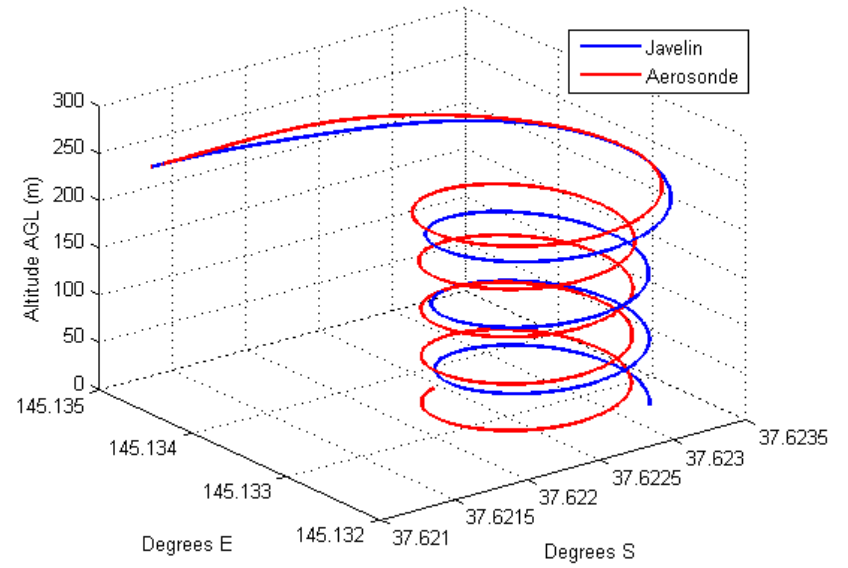

(a) JAVELIN vs. AEROSONDE

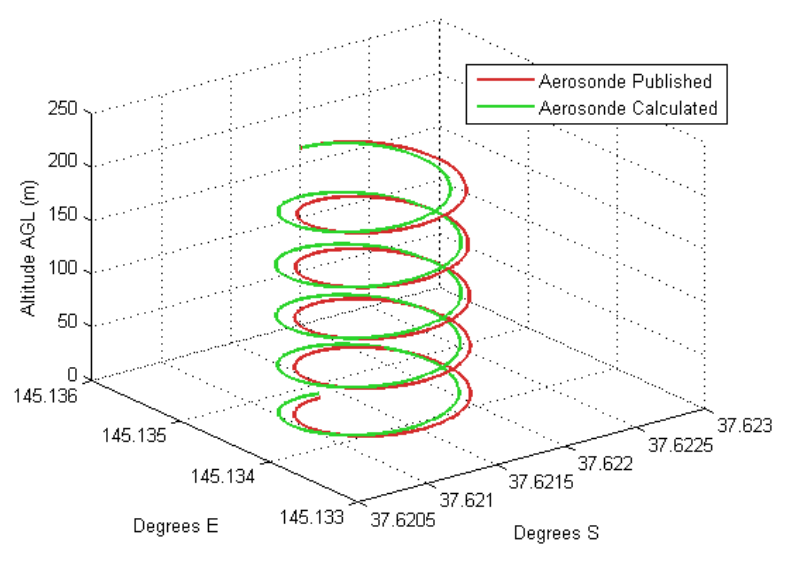

(b) Calculated vs. published AEROSONDE

Fig. 3. Comparison of the simulated trajectories.

\section{Virtual Sensor Simulation}

ADMs are currently being used as a virtual sensor to provide additional information augmenting the navigation sate vector [12] in terms of Position, Velocity and Attitude (PVA) measurements. In current research, the ADM output is being processed either by a centralized EKF (for data fusion with GNSS, MEMS-IMU and VBN) or pre-processed by a dedicated UKF for successive federated UKF/EKF processing with other sensors. The AEROSONDE ADM was used in [13] with a centralized EKF approach and it was found to exceed RNP 1 lateral accuracy requirements after 65 seconds, and precision approach CAT I, CAT II and CAT III requirements after 34, 19 and 16 seconds respectively $[6,13]$. In the attempt to increase the ADM solution validity time, a UKF preprocessing algorithm has been implemented and tested on the AEROSONDE UA as described in [13]. To proceed with an assessment of the JAVELIN ADM as a virtual sensor, an operationally representative flight profile was simulated as shown in Fig. 4. Simulation results showed 
This is the author pre-publication version. This paper does not include the changes arising from the revision, formatting and publishing process. The final paper that should be used for referencing is:

M. Burston, R. Sabatini, R. Clothier, A. Gardi, S. Ramasamy, "Reverse Engineering of a Fixed Wing Unmanned Aircraft 6-DoF

Model for Navigation and Guidance Applications", Applied Mechanics and Materials, vol. 629, pp. 164-169, Trans Tech

Publications, 2014. DOI: 10.4028/www.scientific.net/AMM.629.164

comparable behavior and performance with the AEROSONDE ADM previously tested [6, 13]. In particular, both the JAVELIN and the AEROSONDE cases evidenced that a low-cost VBN/MEMSIMU/GNSS navigation system augmented with UKF pre-filtered ADM (VIGA ${ }^{+}$) can attain position, velocity and attitude accuracies compliant with Category Two (CAT II) precision approach requirements. Additionally, the two ADM virtual sensors performed similarly in terms of precision approach lateral/vertical accuracy and validity time (Table 3).

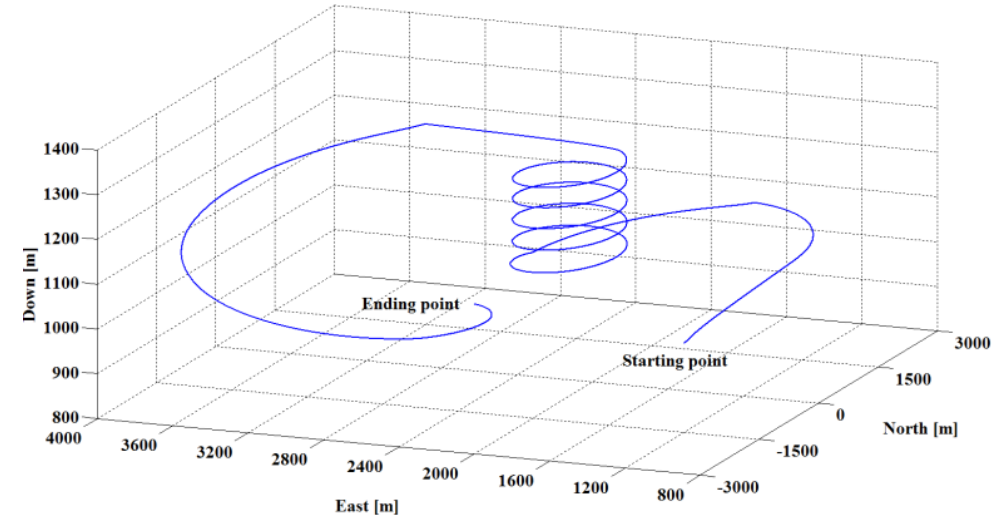

Fig. 4. Simulated 3D trajectory of the JAVELIN UA flight profiles.

Table 3. ADM lateral guidance validity time for JAVELIN and AEROSONDE.

\begin{tabular}{|c|c|c|}
\hline \multirow{2}{*}{ Accuracy threshold } & \multicolumn{2}{|c|}{ ADM validity time [sec] } \\
\hline & JAVELIN VIGA $^{+}$ & AEROSONDE VIGA $^{+}$ \\
\hline CAT I & 92 & 56 \\
\hline CAT II & 37 & 30 \\
\hline CAT III & 18 & 20 \\
\hline
\end{tabular}

\section{Conclusions and Future Work}

The lack of reliable 6-DoF aircraft dynamics models, especially for small UA, can be addressed through after-market development of dynamics models by using reverse engineering techniques. These techniques, combined with implementation of 6-DoF dynamics models as virtual navigation and guidance sensors, potentially provide a low-cost, low-weight/volume option for improving the performance, safety and reliability of small UA [2] in an avionics based integrity augmentation system $[14,15]$. The qualitative validation of the method for determination of aerodynamic parameters was performed on the JAVELIN UA and on the AEROSONDE UA. The 6-DoF models developed for each UA were used to simulate trajectories which were compared against the published 6-DoF data available in the literature. Wind tunnel testing is required to achieve this and to generate reliable engine and propeller data. Flight testing, when conducted, will provide a basis for comparison as well as a method for further improvement of the aerodynamic derivatives through analysis of the aircraft response outside of laboratory conditions. Following the present conceptual validation phase based on simulation activities, actual flight test of the JAVELIN UA will be conducted to verify the performance of the 6-DoF aircraft dynamics model acting as a virtual sensor onboard a small UA. The JAVELIN UA 6-DoF model will be adopted for developing the NextGeneration Flight Management System (NG-FMS) [16] and for Air Traffic Management (ATM) studies [17].

\section{References}

[1] V. Raja and K. J. Fernandes, Reverse Engineering: An Industrial Perspective, London: SpringerVerlag London Limited. (2008) 
This is the author pre-publication version. This paper does not include the changes arising from the revision, formatting and publishing process. The final paper that should be used for referencing is:

M. Burston, R. Sabatini, R. Clothier, A. Gardi, S. Ramasamy, "Reverse Engineering of a Fixed Wing Unmanned Aircraft 6-DoF Model for Navigation and Guidance Applications", Applied Mechanics and Materials, vol. 629, pp. 164-169, Trans Tech Publications, 2014. DOI: 10.4028/www.scientific.net/AMM.629.164

[2] R. Sabatini, S. Ramasamy, A. Gardi and L. Rodriguez, Low-cost Sensors Data Fusion for Small Size Unmanned Aerial Vehicles Navigation and Guidance, International Journal of Unmanned Systems Engineering, 1(3), pp. 16-47. (2013) DOI: 10.14323/ijuseng.2013.11

[3] R. Sabatini, M. Richardson, C. Bartel, T. Shaid and S. Ramasamy, A Low-cost Vision Based Navigation System for Small Size Unmanned Aerial Vehicle Applications, Journal of Aeronautics and Aerospace Engineering, 2(2). (2013) DOI: 10.4172/2168-9792.1000110

[4] R. Sabatini, C. Bartel, A. Kaharkar, T. Shaid, L. Rodriguez, D. Zammit-Mangion and H. Jia, Low-Cost Navigation and Guidance Systems for Unmanned Aerial Vehicles - Part 1: Vision-Based and Integrated Sensors, Annual of Navigation, 19(2), pp. 71-98. (2012) DOI: 10.2478/v10367-0120019-3

[5] R. Sabatini, C. Bartel, A. Kaharkar, T. Shaid and S. Ramasamy, Navigation and Guidance System Architectures for Small Unmanned Aircraft Applications, International Journal of Mechanical, Industrial Science and Engineering, 8(4), pp. 733-752. (2014)

[6] Unmanned Dynamics LLC, AeroSim User's Guide Version 1.2, Hood River, Oregon, USA.

[7] Wikipedia, AAI Corporation AEROSONDE, Wikimedia Foundation. (2013) [Online]. Available: http://en.wikipedia.org/wiki/AAI_Corporation_Aerosonde. [Accessed $29^{\text {th }}$ Sept. 2013]

[8] CREAFORM, Technical Specifications: Handyscan Handheld 3D Scanner, CREAFORM. (2013) [Online]. Available: http://www.creaform3d.com/en/metrology-solutions/products/portable3d-scanner/technical-specifications-handyscan-3d. [Accessed $29^{\text {th }}$ Sept. 2013]

[9] M. Burston, R. Sabatini, A. Gardi and R. Clothier, Reverse Engineering of a Fixed Wing Unmanned Aircraft 6-DoF Model Based on Laser Scanner Measurements, in proceedings of IEEE International Workshop on Metrology for Aerospace 2014, Benevento, Itay. (2014)

[10] M. Napolitano, Aircraft dynamics: from modeling to simulation, Hoboken, NJ: John Wiley \& Sons, Inc. (2012)

[11] A. Deperrois, "XFLR5". [Online]. Available: http://www.xflr5.com/ xflr5.htm. [Accessed $29^{\text {th }}$ Sept. 2013]

[12] R. Sabatini and G. B. Palmerini, RTO AGARDograph AG-160 Vol. 21: Differential Global Positioning System (DGPS) for Flight Testing, NATO Science and Technology Organization, 2008.

[13] R. Sabatini, F. Cappello, S. Ramasamy, A. Gardi and R. Clothier, An Innovative Navigation and Guidance System for Small Unmanned Aircraft using Low-Cost Sensors, Aircraft Engineering and Aerospace Technology (Special Issue: AEROTECH V, Kuala Lampur, Malaysia). (2014)

[14] R. Sabatini, T. Moore and C. Hill, A New Avionics-Based GNSS Integrity Augmentation System: Part 1 - Fundamentals, Journal of Navigation, 66(3), pp. 363-384. (2013) DOI: $10.1017 / \mathrm{S} 0373463313000027$

[15] R. Sabatini, T. Moore and C. Hill, A New Avionics-Based GNSS Integrity Augmentation System: Part 2 - Integrity Flags, Journal of Navigation, 66(4), pp. 501-522, (2013) DOI: $10.1017 / \mathrm{S} 0373463313000143$

[16] S. Ramasamy, R. Sabatini, A. Gardi and Y. Liu, Novel Flight Management System for Real Time 4-Dimensional Trajectory Based Operations, in proceedings of AIAA Guidance, Navigation \& Control Conference, Boston, Massachusetts, USA. (2013) DOI: 10.2514/6.2013-4763

[17] A. Gardi, R. Sabatini, S. Ramasamy and K. de Ridder, 4-Dimensional Trajectory Negotiation and Validation System for the Next Generation Air Traffic Management, in proceedings of AIAA Guidance, Navigation \& Control Conference, Boston, Massachusetts, USA. (2013) DOI: $10.2514 / 6.2013-4893$ 